\title{
Paleomagnetic investigation of the great egyptian pyramids
}

口 Igor Túnyi ${ }^{1}$, Ibrahim A. El-hemaly ${ }^{2}$ - DOI: 10.1051/epn/2012604

I 'Geophysical Institute SAS - Bratislava - tunyi@up.upsav.sk

I 'National Research Institute of Astronomy and Geophysics - Cairo - elhemaly_1@yahoo.co.uk

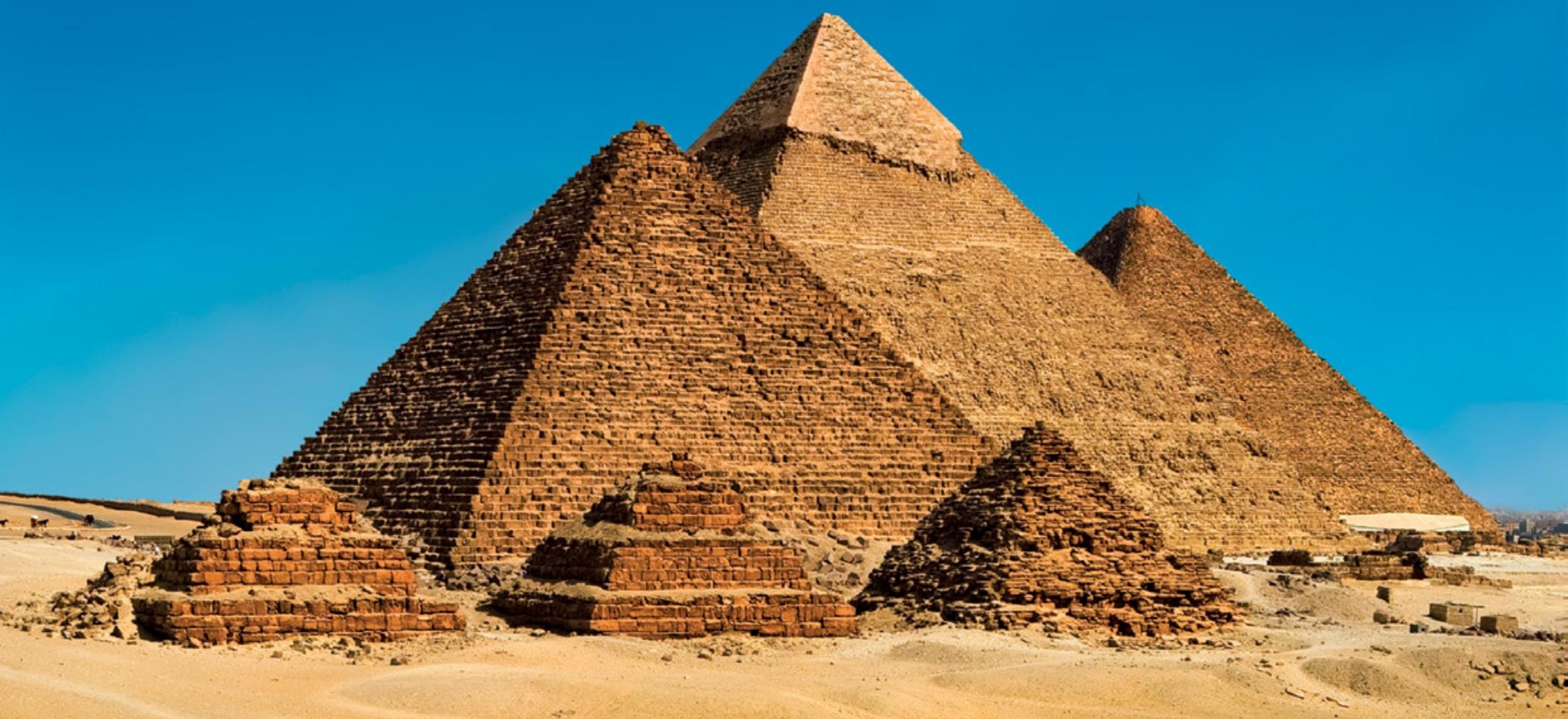




\section{The great Egyptian pyramids present one of the greatest mysteries of mankind. Despite continuous scientific investigations, these monuments of antiquity still hide many secrets. Unresolved remains the question regarding the material and building technology of the pyramids. There are two opposing views.}

I:

$t$ is generally accepted that they were constructed from cubic blocks of natural limestone, transported from the quarries in the surroundings of Giza. However, during the last century, several authors advocated the supposition that the cubes might have been produced from the mud of the Nile in situ in special wooden containers filled with a liquid called natron, a salt mixture from dry lake beds. In this way, a rocky concrete, called geopolymer, had been produced which, after having dried out, has properties similar to the natural limestone.

This technology has been described in detail by Demortier [1]. If we assume that this method is plausible, then paleomagnetism can be one of the instruments to verify this.

\section{Method}

Each rock, volcanic or sedimentary, acquires its remanent magnetic polarization during its formation. Ferromagnetic grains fix their magnetic orientation (volcanites when they pass through the Curie point and sediments during the sedimentation process) in the geomagnetic field. The resulting direction of the rock magnetic moment is parallel to the direction of geomagnetic field. The same process is active during the production of thermally produced artifacts from rock material like bricks, ceramics, etc.

In paleomagnetic research, rock samples are collected and the direction of their characteristic magnetic polarization is determined. Such paleomagnetic records find applications particularly in geology. However, since rocks or rocky artifacts may have been affected during their history by different agents (e.g., heat, pressure, chemical reactions), a parasitic secondary magnetic polarization might have been acquired, and hence the so-called magnetic cleaning of the samples is necessary. During this cleaning, the secondary polarization is eliminated and the original magnetic characteristic is recovered. Thermal demagnetization is the most frequently used cleaning technique, in which the sample is step-wise heated to a temperature above the Curie temperature of any ferromagnetic material.

The sample is subsequently cooled in a nonmagnetic environment after each thermal increment $\left(e . g ., 50^{\circ} \mathrm{C}\right)$ and the direction and intensity of its magnetic polarization is recorded. Soft secondary magnetic polarization is usually relieved at lower temperatures, while the hard original polarization undergoes demagnetization only at higher temperatures. In addition to the direction and intensity of the magnetic polarization, the magnetic susceptibility is also recorded. If the magnetic susceptibility does not change during demagnetization, then no phase change of the magnetic material has occurred (rock keeps its original composition). By contrast, rapid changes in susceptibility with temperature indicate that the rock material underwent phase changes, and further measurement is irrelevant.

Our paleomagnetic investigation of the two great Egyptian pyramids, Kufu and Khafre, is based on the assumption that if the blocks were made in situ by the concrete technique [1] described above, then their magnetic moments would all have been parallel, oriented approximately in the north-south direction. However, if the pyramids were constructed from blocks transported from the nearby quarries, having been rotated randomly during transport and construction, then the directions of their magnetic moments would be oriented randomly.

\section{Results}

Samples were collected from 6 locations in the two pyramids, 3 from Khufu and 3 from Khafre (Fig. 1). Demortier [1] admits that even if the concrete technique was used,

v Fig. 1: Sampling locations on the Khufu and Khafre pyramids and in the adjacent quarry. $\mathrm{N}=$ geographic North. Red arrows indicate the directions of magnetic polarization vectors (paleodirections) in the samples taken from locations 1,2 and 5 .

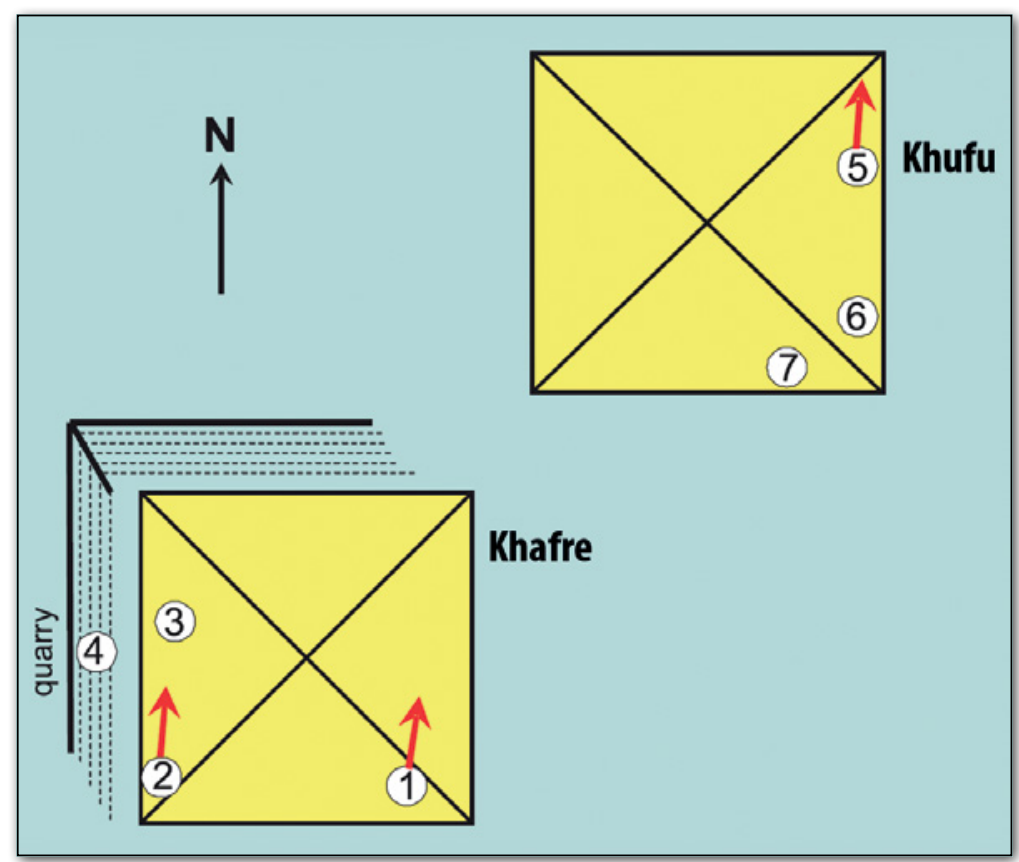

$\varangle$ Egypt. Cairo - Giza General view of pyramids from the Giza Plateau (from left - the Pyramid of Menkaure, Mykerinos; Khafre, Chephren and Chufu, Cheops known as the Great small pyramids popularly known as Queens' Pyramids on front side). The Pyramid Fields from Giza to Dahshur are on the UNESCO World Heritage List. CiStockPhoto Pyramid and three 
it is most probable that there might be a combination of artificial and natural blocks. We find (Fig. 1) that the Khafre pyramid lies partly in a small quarry forming the base of the pyramid. It is

The results of this project have proved the usefulness of paleomagnetic methods highly probable that blocks cut from this quarry were used to build the pyramids. This opinion was visually verified. The rock material from the quarry is very similar to the blocks material in the adjacent parts of pyramid. Therefore, in addition to the 3 sampling positions described above, other samples from the quarry at the position marked 4 in Fig. 1 were collected for comparison. Note that at least two samples were obtained from each position.

v Fig. 2: Thermal demagnetization of the samples from locations 1, 2, 3 and 4(see Fig. 1). $\mathrm{N}=$ geographic North. The dots inside the big circles indicate the directions of the magnetic polarization vectors in the samples (see the main text) after heating to the temperature values specified by the numbers. Curve $\mathrm{J}_{\mathrm{T}} / \mathrm{J}_{0}$ : magnetic polarization $\left(\mathrm{J}_{\mathrm{T}}\right)$ of the samples, heated to temperature $\mathrm{T}$, divided by its initial value $\left(J_{0}\right)$. Curve $\kappa_{\mathrm{T}} / \kappa_{0}$ : magnetic susceptibility $\left(\kappa_{\mathrm{T}}\right)$ of the samples after heating to temperature $T$, relative to its initial value $\left(\kappa_{0}\right)$.

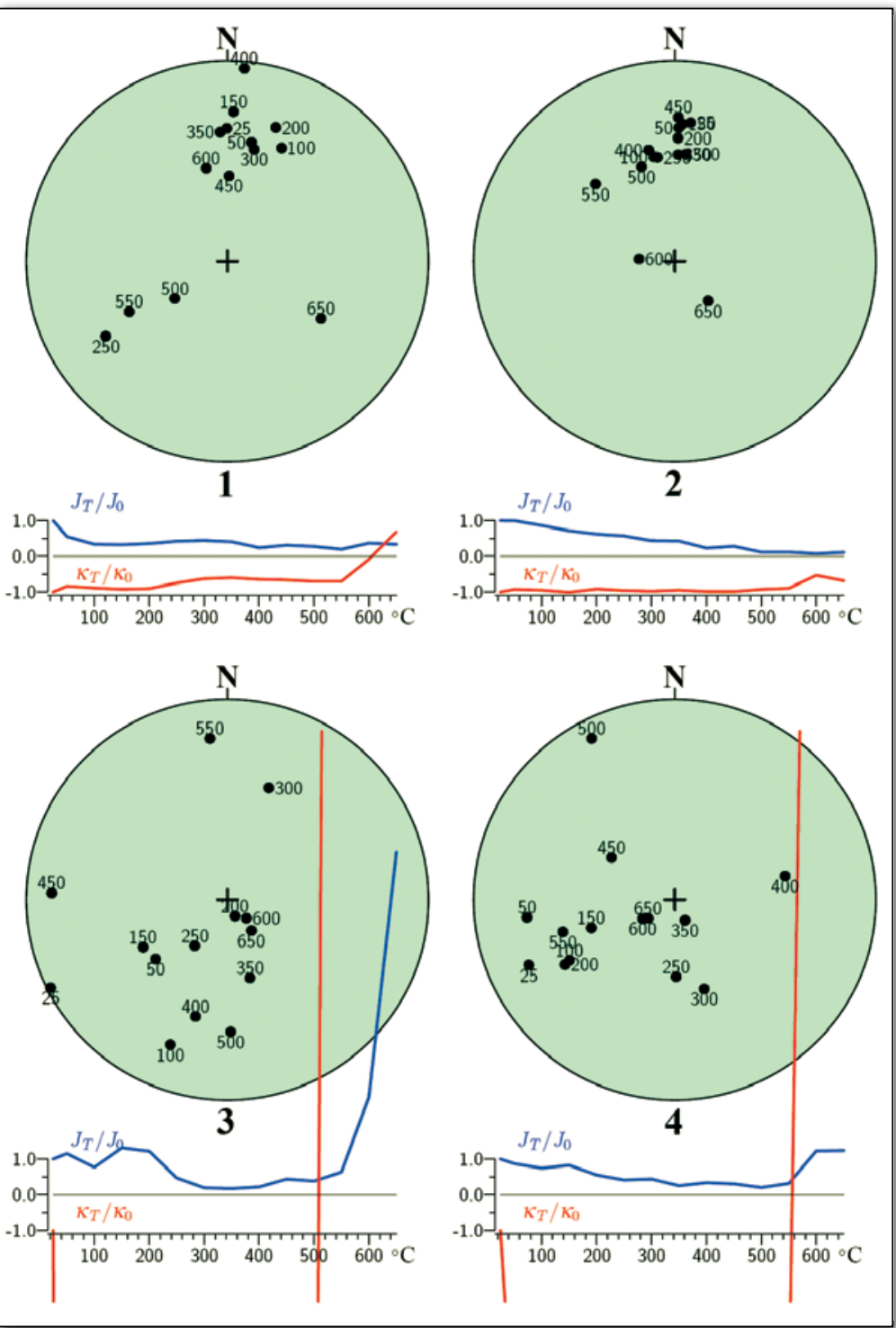

The measurements were performed in the Paleomagnetic Laboratory at the Geophysical Institute of the Slovak Academy of Sciences in Bratislava. The method of thermal demagnetization, together with adequate tests of paleomagnetic stability [2], were applied, and the results are presented in Figs. 2 and 3. Here, the dots represent projections of the tips of the magnetic polarization vectors onto the surface of a unit sphere, with the polarization vectors originating in the centre of the sphere. The big circles represent the projections of the unit sphere onto the horizontal plane. The symbol $\mathrm{N}$ denotes the direction of the true geographic North. The angular differences between direction of the true geographic North and the directions represented by the dots indicate declination. Furthermore, the distances between the centre of the big circle and the dots indicate the inclination of the geomagnetic field which magnetized the rocks in the locations where the sampling took place. (The inclination at the equator of the geomagnetic field is zero; the corresponding dot would lie on the circumference of the big circle. The inclination at the pole of the geomagnetic field is $90^{\circ}$; again the corresponding dot would project onto the centre of the big circle.)

The curves represent the thermal dependence of magnetic polarization $(\mathrm{J})$ and magnetic susceptibility $(\kappa)$ of the samples measured. Both quantities are normalized to their original values and shown in the plots as demagnetizing curves.

In the stereoprojections (Figs. 2 and 3 ) we can see that for samples from locations 1,2 and 5, the north-south direction of their magnetic moments prevails.

We see that the samples from locations 3 and 4 have qualitatively similar demagnetizing curves. In both samples, a steep change in the magnetic susceptibility at about $400^{\circ} \mathrm{C}$ can be seen, which suggests that the samples contain the same magnetic material. It is impossible to identify any preferred direction from the range of directions in their magnetic polarizations. As described above, the material from locations 3 and 4 are very similar in nature. Together with the similarity in their magnetic characteristics, we may infer that the block from location 3 has its origin in the quarry near the pyramid (Fig. 1).

The samples from locations 1,2 and 5 exhibit the same demagnetizing characteristics and their paleodirections (the average direction of magnetic polarization vectors) are nearly identical, as seen from Figs 2 and 3. This indicates that the blocks from which these samples were cut have identical carriers of magnetism and probably also identical rock material - perhaps the artificial limestone (geopolymer) prepared by the concrete technique described above. The paleodirections of the samples considered are presented in Fig. 4. In principle, they correspond to the declination and inclination of the geomagnetic field in Egypt. 


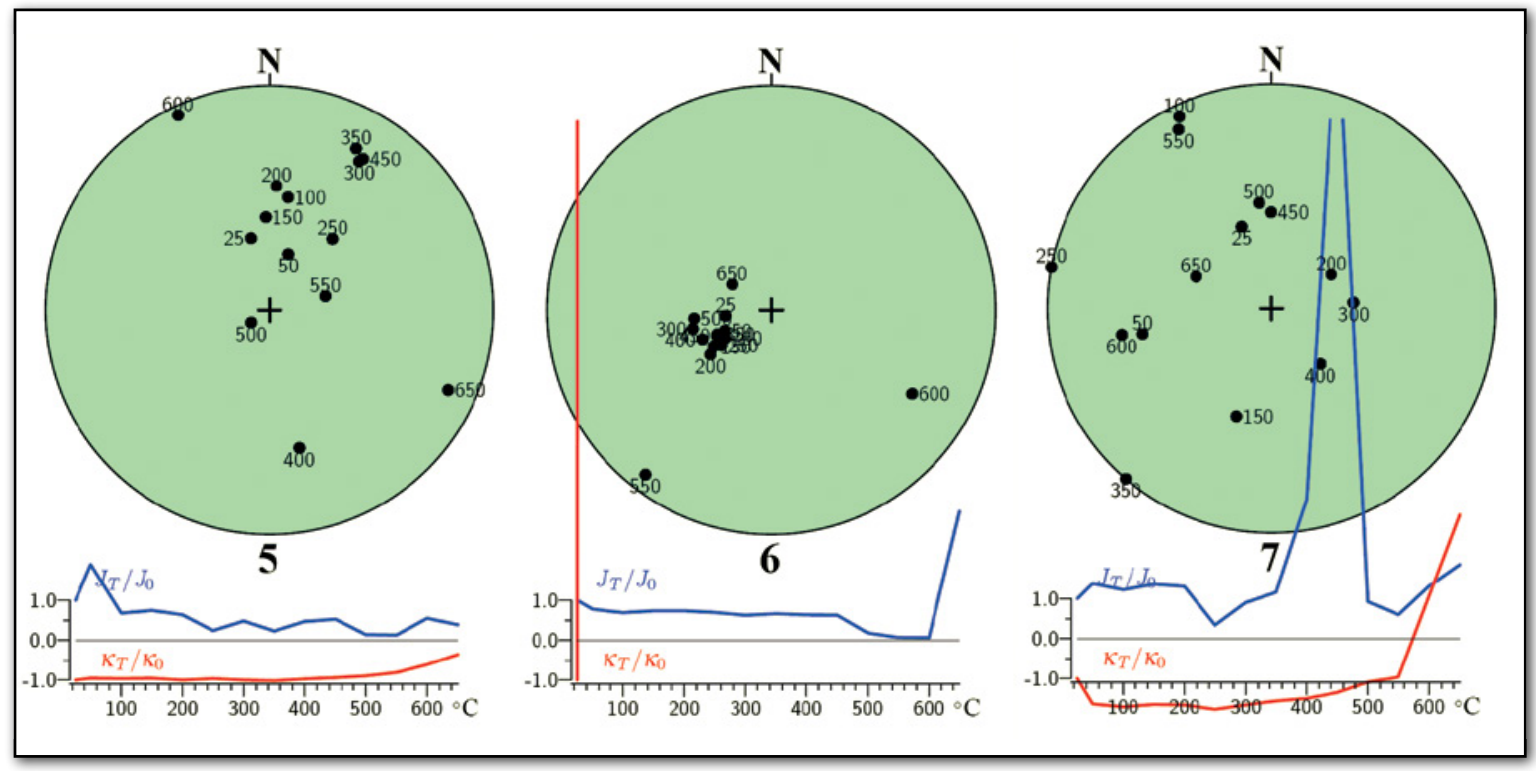

The sample from location 7 shows demagnetizing characteristics similar to those from locations 3 and 4 . This suggests that the block from which the sample was taken comes also from the quarry near the Khafre pyramid. Although the sample from location 6 allows one to determine the preferred magnetic direction, the rapid change in magnetic susceptibility at low temperatures (see Fig. 3) makes this paleodirection irrelevant. Therefore, we conclude that the rock material of the block from location 6 is different from that of blocks at other locations.

The arrows in Fig. 1 indicate the calculated paleodirections.

\section{Conclusion}

The aim of paleomagnetic investigation of the rock material of the great Egyptian pyramids, Khufu and Khafre, was to find out the directions of the magnetic polarization vectors of their building blocks. This is one of the possible ways to verify the hypothesis according to which the blocks were produced in situ by a concrete technique. The analysis of a limited set of paleomagnetic samples provided the following results. The paleodirections of three sampling locations ( 2 from Khafre and 1 from Khufu pyramid) exhibit the common north-south orientation, suggesting that they may have been produced in situ by a concrete technique. The block from one sampling location of the Khafre pyramid is of natural limestone and evidently comes from the adjacent quarry. It is likely that the block from one sampling position of the Khufu pyramid comes also from the same quarry. Finally, we conclude that even if the concrete technique was used, the pyramids were constructed from a mixture of natural and artificial limestone blocks.
The results of this project have proved the usefulness of paleomagnetic methods. A more thorough investigation would provide further insight into the building material and the construction technology of the great Egyptian pyramids.

\section{About the Authors}

Igor Túnyi is a geophysicist and paleomagnetist at the Geophysical Institute of the Slovak Academy of Sciences, Dúbravská cesta 9, 84528 Bratislava, Slovakia.

Ibrahim A. El-hemaly is an applied and environmental geophysicist at the National Research Institute of Astronomy and Geophysics, P.O. Box 11722 Helwan, Cairo.

\section{References}

[1] G. Demortier, Revisiting the Construction of the Egyptian Pyramids, Europhysics News 40/1, 27.

[2] M.W. McElhinny and P.L. McFadden, Paleomagnetism, Academic Press, London, 385.

$\checkmark$ Fig 4: Directions of the characteristic magnetic polarization vectors of samples from locations 1, 2 and 5 (see Fig. 1). $\mathbf{N}=$ geographic North. Dots: directions of specimens from the same location. The cross symbols represent the mean directions. The blue circle indicates the region in which the polarization vector projection falls with $95 \%$ probability.

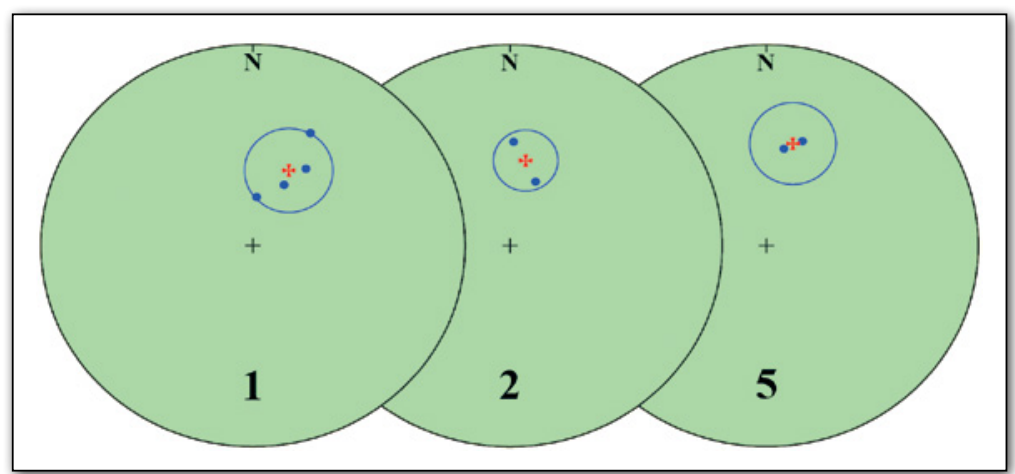

